\title{
Precessing jets from a moving source and bright $X$-ray filaments in galaxy clusters
}

\author{
M. Rodríguez-Martínez ${ }^{1,3}$, P. F. Velázquez ${ }^{2}$, L. Binette ${ }^{3}$, and A. C. Raga ${ }^{2}$ \\ ${ }^{1}$ Harvard-Smithsonian Center for Astrophysics, MS-83, 60 Garden Street, Cambridge, MA 02138, USA \\ e-mail: mario@head.cfa.harvard.edu \\ 2 Instituto de Ciencias Nucleares, Ciudad Universitaria, Apartado Postal 70-543, CP 04510, \\ México D.F., México \\ e-mail: [pablo,raga] @nucleares.unam.mx \\ 3 Instituto de Astronomía, Universidad Nacional Autónoma de México, Ciudad Universitaria, Apartado Postal 70-248, CP 04510, \\ México D.F., México \\ e-mail: binette@astroscu.unam.mx
}

Received 7 May 2005 / Accepted 3 October 2005

\section{ABSTRACT}

We present hydrodynamical calculations carried out with the 3D YGUAZÚ-A code for a precessing jet model that is interacting with a plane parallel wind. This scenario describes an extragalactic jet in which the jet source is in motion with respect to the surrounding intra-cluster medium. From the numerical results, synthetic emission maps and spectra in X-ray band were obtained. We compare these predictions with observations of the radio jets emanating from the radio-galaxy 4C 26.42 (in the Abell 1795 galaxy cluster). We find that the general morphology of the radio jets can be described by a point-symmetric precessing jet system interacting with a plane parallel wind (i.e., the intra-cluster medium flowing past the galaxy). We also find that our synthetic X-ray emission maps reproduce the observed large scale structures (with sizes on the order of tens of $\mathrm{kpc})$.

Key words. hydrodynamics - methods: numerical - galaxies: jets - X-rays: galaxies: clusters

\section{Introduction}

Extragalactic jets show three basic types of morphological structures (observed in radio-continuum, Zaninetti \& Van Horn 1988): the linear shape, the tail or " $C$ " shape and the " $Z$ " (or "S") shape. The "C" and "Z" shapes are usually interpreted as being caused by either the relative motion of the radio galaxy with respect to the ambient medium ("C-shape") or by the precession of the jet direction ("Z") A combination of both effects is probably present in most objects, but one of them is usually starkly dominant. The orientation of the observer relative to the jet direction also plays an important role.

Several authors have studied extragalactic jets by means of numerical simulations. We can mention the MHD model developed by Pietrini \& Torricelli-Ciamponi (1992) and TorricelliCiamponi \& Pietrini (1993), where they analyze the formation of structures along the jet axis. Cox et al. (1991) carried out 3D numerical simulations of precessing jets in order to explain the formation of hot-spots and the general morphology observed in extragalactic radio sources. Ferrari (1998) gives a thorough review of the modeling of extragalactic jets. Interestingly, in the arena of stellar jets, the "S-shape" has likewise been observed and modeled by invoking precession, such as in HH 34
(Masciadri et al. 2002, and references therein) or in the protoplanetary nebula Hen 3-1475 (Velázquez et al. 2004a, and references therein).

As examples of extragalactic jet with "S" shapes, we can mention the jet system from the radio source 4C 26.42 (in the Abell 1795 galaxy cluster, van Breugel et al. 1984; and Ge \& Owen 1993), the radio galaxies 3C 294 (Crawford et al. 2003), 3C 334 (Kellermann \& Owen 1988), and 4C 29.47 (Condon $\&$ Mitchell 1984). These jets are immersed in an intra-cluster medium (ICM) and their radio lobes can be asymmetric due to the motion of the jet source. This fact was reported by Hill et al. (1988) for the case of the radio source 4C 26.42, which is in motion within the gravitational potential of the Abell 1795 cluster, with a peculiar radial velocity of $365 \mathrm{~km} \mathrm{~s}^{-1}$. However, Oegerle \& Hill (1994) later revised this measurement and favored a lower value of $150 \mathrm{~km} \mathrm{~s}^{-1}$.

While the properties of extragalactic jets can be studied with radio-continuum observations, the ICM in which these objects are propagating can be detected in $\mathrm{H} \alpha$ or $\mathrm{X}$-ray emission. Recently, a new generation of X-ray satellites (with the Advanced Camera for Imaging and Spectroscopy of CHANDRA, and XMM-Newton) have obtained images and 
spectra with good spatial and spectral resolution (respectively) of galaxy clusters containing radio jets.

$\mathrm{X}$-ray and radio observations provide evidence of jet-ICM or ISM interactions. Zanni et al. (2003) and Reynolds et al. (2001) (Rizza et al. 2000) carried out 2D (axisymmetric) and 3D numerical simulations, respectively, in order to analyze this interaction and its observational consequences. They report two main regimes for the jet-ICM interaction. In the first one, the jet cocoon expands into the ICM supersonically, giving a shell-like morphology in the X-ray emission that surrounds the radio-jets. The second regime is characterized by subsonic cocoon expansion. In this case, the X-ray emission is strong at the head of the jet, while the sides of the cocoon practically disappear. Zanni et al. (2003) found that the main parameter determining the appropriate regime is the jet kinetic power (or ram pressure).

The models listed in the preceding paragraph predict shelllike or partial shell-like morphology for the X-ray emission from the jet-ICM interaction. However, several authors have reported that the $\mathrm{X}$-ray emission of some galaxy clusters has bright filamentary structures embedded in the diffuse emission (Fabian et al. 2001, for the Abell 1795 case; Crawford et al. 2003, for 3C 294).

In this paper, we explore the possibility that the jet-ambient gas interaction leads to a filamentary morphology in X-ray, as a result of two processes: the precession of the point-symmetric jets and the displacement of the central galaxy with respect to the intra-cluster medium. We present numerical simulations carried out with the 3D YGUAZÚ-A code (Raga et al. 2000), which includes these two effects.

This work is organized in the following way. In Sect. 2 we present the model employed in our simulations, giving the initial conditions and describing the assumptions that have been made. We also explain how the derived X-ray emission is obtained from our numerical results. In Sect. 3, we present both the hydrodynamical simulations and the predicted X-ray emission maps. In Sect. 4 we analyze our numerical results and compare them with observations, and finally in Sect. 5 we give our final discussion.

\section{Description of the model}

In order to model the interaction of an extragalactic radio-jet with the surrounding environment, we consider a model consisting of a point-symmetric, precessing jet system interacting with a plane-parallel wind.

We consider this system with a fixed jet velocity, where the precession is characterized by a semi-opening angle $\alpha$ and precession period $\tau_{\mathrm{p}}$. The adopted parameters are listed in Table 1 . An important aspect to consider is the " $\mathrm{S}$ " morphology of some radio-jets exhibits some degree of asymmetry between the two radio lobes. This asymmetry can be produced by the motion of the $\mathrm{cD}$ galaxy with respect to the surrounding intra-cluster medium. Velocities in the range $[150,350] \mathrm{km} \mathrm{s}^{-1}$ have been reported from observations by Oergerle \& Hill (1994) and Hill et al. (1988) for the case of the 4C 26.42 radio-jet. This effect has been explicitly taken into account in our models, although for computational purposes, the galaxy is deemed stationary
Table 1. Input parameters of model I.

\begin{tabular}{ll}
\hline \hline Parameter & Value \\
\hline$x$ size & $1.5 \times 10^{23} \mathrm{~cm}$ \\
$y$ size & $1.5 \times 10^{23} \mathrm{~cm}$ \\
$z$ size & $3.0 \times 10^{23} \mathrm{~cm}$ \\
ambient density, $n_{w}$ & $0.1 \mathrm{~cm}^{-3}$ \\
ambient temperature, $T_{w}$ & $6 \times 10^{7} \mathrm{~K}$ \\
wind velocity, $v_{w}$ & $3 \times 10^{7} \mathrm{~cm} \mathrm{~s}^{-1}$ \\
wind entrance angle, $\beta$ & $60^{\circ}$ \\
jet density, $n_{\mathrm{j}}$ & $5 \times 10^{-3} \mathrm{~cm}^{-3}$ \\
jet temperature, $T_{\mathrm{j}}$ & $6 \times 10^{7} \mathrm{~K}$ \\
jet velocity, $v_{\mathrm{j}}$ & $10^{9} \mathrm{~cm} \mathrm{~s}^{-1}$ \\
precession period, $\tau_{\mathrm{p}}$ & $10^{15} \mathrm{~s}$ \\
half precession angle, $\alpha$ & $20^{\circ}$ \\
\hline
\end{tabular}

within the $3 \mathrm{D}$ grid, while it is the intra-cluster medium which is moving at a velocity of $300 \mathrm{~km} \mathrm{~s}^{-1}$.

\subsection{Initial conditions and assumptions}

In order to determine physical parameters of the extragalactic jets and the ICM, we considered the observational results of van Breugel et al. (1984), Fabian et al. (2001), and Ettori et al. (2002).

Our 3D numerical simulations were carried out with the YGUAZÚ-A code (Raga et al. 2000), which integrates the gasdynamical equations with a second order accurate technique (in time and space) that employs the flux-vector splitting method of van Leer (1982). Our numerical simulations use a 5-level, binary adaptive grid with a maximum resolution of $5.86 \times$ $10^{20} \mathrm{~cm}$, in a computational domain of $(1.5,1.5,3.0) \times 10^{23} \mathrm{~cm}$ along the $x$-, $y$ - and $z$-axis, respectively. The jets are injected at $x=y=7.5 \times 10^{22} \mathrm{~cm}$ and $z=1.5 \times 10^{23} \mathrm{~cm}$ (i.e. at the center of the computational domain). An injection jet velocity $v_{\mathrm{j}}=10^{9} \mathrm{~cm} \mathrm{~s}^{-1}$, a precession angle $\alpha=20^{\circ}$, and a precession period $\tau_{\mathrm{p}}=10^{15} \mathrm{~s}$ were considered. The jet precession cone axis is aligned with the $z$-axis.

For the intra-cluster medium, the temperature and density were fixed at $6 \times 10^{7} \mathrm{~K}$ and $0.1 \mathrm{~cm}^{-3}\left(2.16 \times 10^{-25} \mathrm{~g} \mathrm{~cm}\right)$, respectively (based on CHANDRA X-ray observations, Ettori et al. 2002). Also, the intra-cluster wind has a velocity of $3 \times 10^{7} \mathrm{~cm} \mathrm{~s}^{-1}$, forming an angle of $60^{\circ}$ with respect to the $x$-axis. For the jets we adopted a temperature of $6 \times 10^{7} \mathrm{~K}$ and density of $5 \times 10^{-3} \mathrm{~cm}^{-3}\left(1.1 \times 10^{-26} \mathrm{~g} \mathrm{~cm}^{-3}\right)$. The initial diameter and length of the jet were of $6 \times 10^{21} \mathrm{~cm}$ (or 10 pixels) and $4 \times 10^{21} \mathrm{~cm}$ (or 7 pixels), respectively. The jet and environmental parameters are summarized in Table 1.

The version of the YGUAZÚ-A code that we have used does not include radiative losses. This simplification is certainly acceptable, since we find that the typical dynamical time $\tau_{\mathrm{d}}=4.7 \mathrm{Myr}$, which characterizes our calculations, is much shorter than the cooling time scale of 127 Myr. Hereafter, we will call the model described in this subsection "model I".

Our jet has a Mach number of $\sim 20$, high enough that the jet pressure does not play an important role. Also, the ram pressure of the jet material is an order of magnitude higher than the 
environmental pressure, so that the jet is not "chocked", and it propagates supersonically (see Fig. 12 of Zanni et al. 2003).

\subsection{Other parameter values}

In order to check how sensitive our model I is to changes of input parameters, we explored two other models. In model II, we chose a precession period that is five times shorter $\left(\tau_{\mathrm{p}}=\right.$ $1.1 \times 10^{14} \mathrm{~s}$ ) than in model I, keeping constant the other input parameters. In model III, we instead changed the incidence angle of the plane-parallel wind to $90^{\circ}$; i.e. the plane-parallel wind is actually moving in the $-z$ direction.

\subsection{Simulated $X$-ray maps and spectra}

From our numerical results, it is possible to obtain X-ray emission maps and also X-ray spectra through line-of-sight integrations. In this way, the numerical results can be compared directly with X-ray observations from satellites such as CHANDRA. Such comparisons have recently been made for other flows by Raga et al. (2001, for the case of the Arches cluster) and Velázquez et al. (2004b, for the X-ray emission of the supernova remnants W44 and G296.5+10.0).

To calculate the emission coefficient, $j_{v}(n, T)$, we used the CHIANTI ${ }^{1}$ atomic database and its associated IDL software (Dere et al. 2001; Young et al. 2003). We then generated the synthetic X-ray emission coefficient (integrated over the energy range of interest) as a function of temperature $T$ (at a reference density of $1 \mathrm{~cm}^{-3}$ ) and then scaled it using the $j \propto n^{2}$ low density regime density dependence. We assumed that the gas is in coronal ionization equilibrium. For calculating X-ray emission, we used the Mazzota et al. (1998) coronal ionization equilibrium and the Anders \& Grevesse (1989) solar abundances.

From our numerical models, we obtained synthetic emission maps corresponding to the soft $([0.3-1.8] \mathrm{keV})$ and hard $([1.8-7] \mathrm{keV})$ energy bands of the CHANDRA satellite. For the simulated spectra, which cover the whole energy band $([0.3-7] \mathrm{keV})$, we considered the effects of interstellar absorption using the absorption curve of Morrison \& McCammon (1983) and integrated the emission coefficient along either the $y$-axis ( $x z$ projection) or the $x$-axis ( $y z$ projection).

\section{Results}

\subsection{Hydrodynamical results}

For analyzing the characteristics of the interacting jet-wind model, the temporal evolution of the density distribution is shown in Fig. 1. These maps are $y=0$ cuts, and they correspond to the density distribution in the $x z$ plane. The density scale is given in units of $\mathrm{g} \mathrm{cm}^{-3}$. A time interval $\Delta t=24.2 \mathrm{Myr}$ separates the sequence of panels displayed in Fig. 1, spanning

\footnotetext{
1 The CHIANTI database and its associated IDL procedures are freely available at: http://wwwsolar.nrl.navy.mil/chianti.html, http://www. arcetri.astro.it/science/chianti/ chianti.html,

http://www.damtp.cam.ac.uk/users/astro/chianti/ chianti.html
}

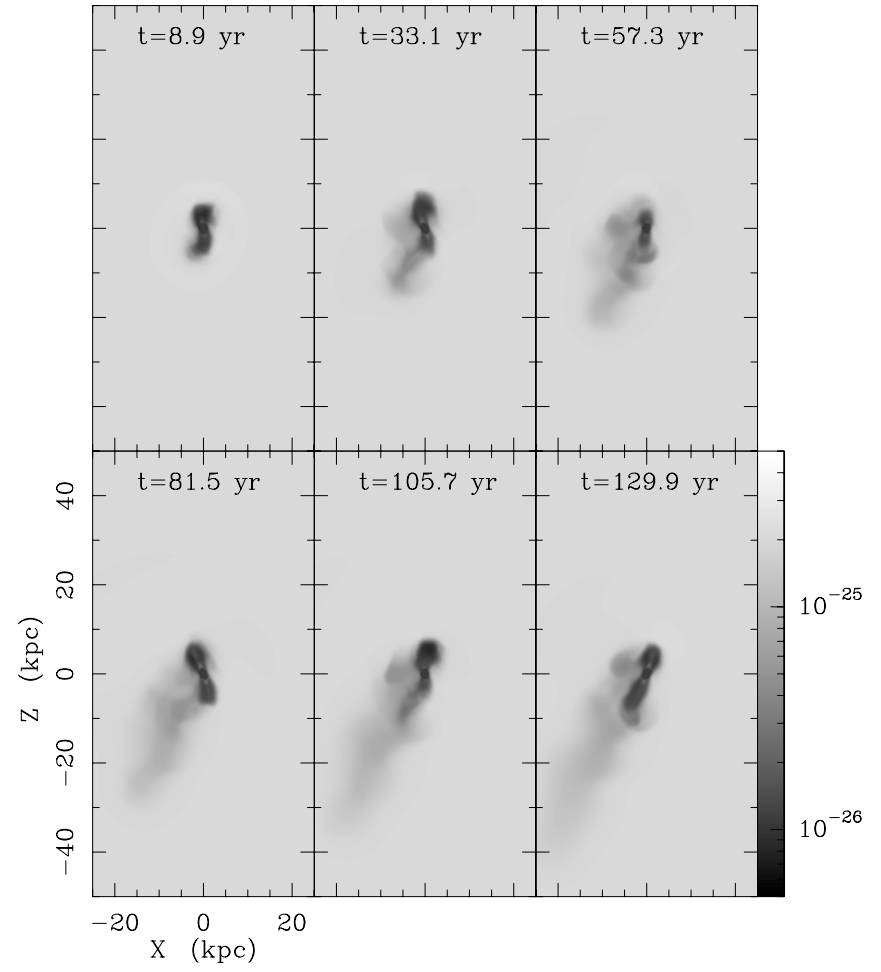

Fig. 1. Time evolution of the density stratification in the $y=0$ plane, for the jet-wind interacting model. Integration times ranges from 8.9 to $130 \mathrm{Myr}$. The logarithmic gray-scale for the density is given by the bar next to the bottom-right panel (in units of $\mathrm{g} \mathrm{cm}^{-3}$ ). The $x$ - and $z$-axes are labeled in units of kpc. Note that darker areas correspond to lower density regions.

a total temporal evolution of $130 \mathrm{Myr}$. A long wake can be observed in Fig. 1 as a result of the interaction between the precessing jets and the plane-parallel ICM wind. This wake has low-density gas, which is swept up by the ICM wind. The density of the wake is $\sim 5 \times 10^{-26} \mathrm{~g} \mathrm{~cm}^{-3}$, i.e. five times lower than its surrounding environment due to the mixing of the shocked jet gas (by the Mach disk) with the denser ICM gas, which has been shocked by the jet bow shock. The jet axis is continously varying its inclination with respect to the velocity of the incident ICM wind. At smaller incident ICM wind-jet axis angles, the collision becomes stronger than in the case with larger ICM wind-jet axis angles; i.e. the ICM wind is actually hitting the cocoon side. This produces small differences in the density of the shocked ICM gas, which is given by:

$\frac{\rho_{2}}{\rho_{1}}=\frac{(\gamma+1) M_{1}^{2}}{(\gamma-1) M_{1}^{2}+2}$

where $\gamma$ is the specific heat ratio, $M_{1}$ is the shock Mach number, and $\rho_{2}$ and $\rho_{1}$ are the shocked and unshocked ICM densities, respectively. For strong shocks, we have $M_{1} \gg 1$, and the density ratio (see Eq. (1)) is approximately equal to 4 (in the monoatomic gas case), while it is slightly smaller than this value, when the shocks have $M_{1} \simeq 1$. Because of this and after the mixing with the low density shocked jet gas, the wake has a knotty structure; and its width is variable when this shocked ICM-jet gas is swept up and pushed behind by the incident flow. 


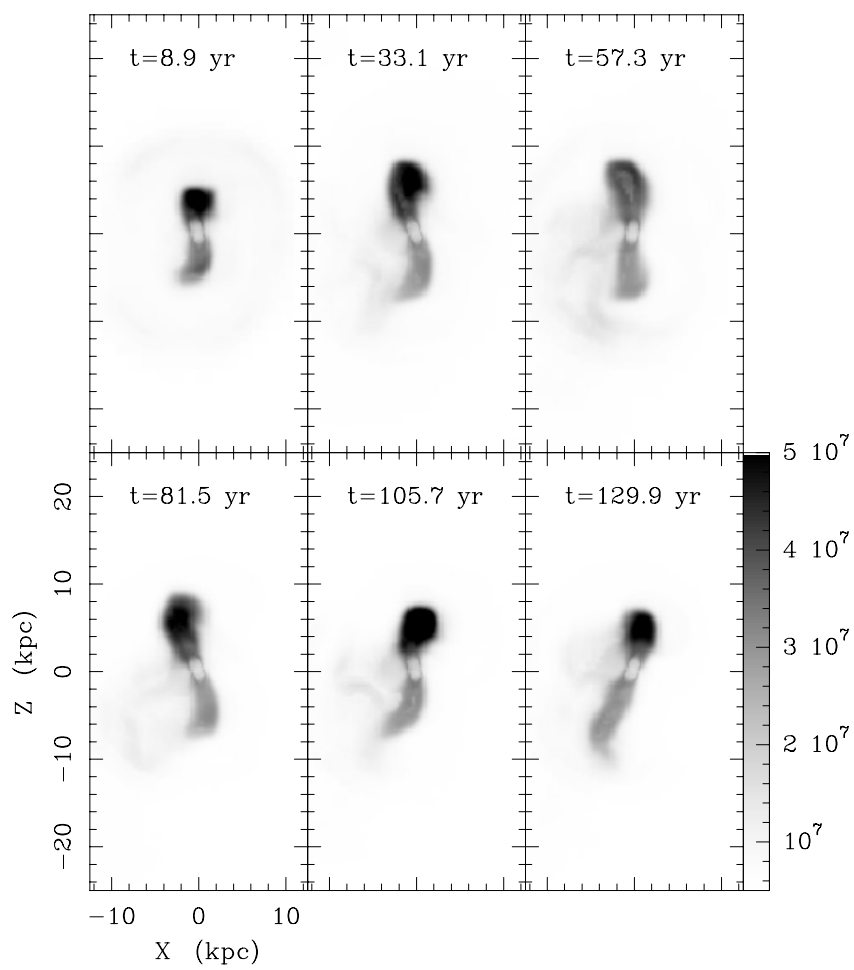

Fig. 2. Temporal evolution of the temperature averaged along lines of sight. Each $x z$ projection map was obtained by integrating the temperature along the $y$ axis. High-temperature regions (dark gray) reveal the morphology of the antisymmetric jets that are emanating from the central source.

On the other hand, from Figs. 1 and 2, the jet lobes look like as cavities of low density and high temperature gas.

In order to directly compare of the numerical results with the observations, one can generate synthetic emission maps at the desired wavelength or energy band (the X-ray emission maps will be discussed later in Sect. 3.2). We could not calculate radio emission maps because the magnetic field is not included in our description. Nevertheless, it is possible to use other tracers for the jet structure. Radio emission from extragalactic jets is due to synchrotron emission (i.e., of nonthermal origin), which is enhanced in sites of strong shock waves. These sites have high temperatures, hence maps of the temperature integrated along lines-of-sight are expected to be good tracers of the radio-jet structure. Figure 2 shows the time evolution of the average integrated temperature from time $t=8.9 \mathrm{Myr}$ to $t=130 \mathrm{Myr}$. High-temperature sites reveal the jet morphology. In this figure we notice an asymmetry between the upper and lower lobe that is due to the wind-jet interaction. The characteristic sizes of these lobes are $10 \times 4 \mathrm{kpc}$ (or $50 \times 20$ pixels $)$.

\subsection{Synthetic $X$-ray emission maps}

Following the procedure described in Sect. 2.3, we derive $\mathrm{X}$-ray emission maps by integrating the X-ray emission coefficient along the selected line-of-sight. In Fig. 3 we display the simulated X-ray maps for model I for two different projections on the plane of the sky. The upper left panel shows

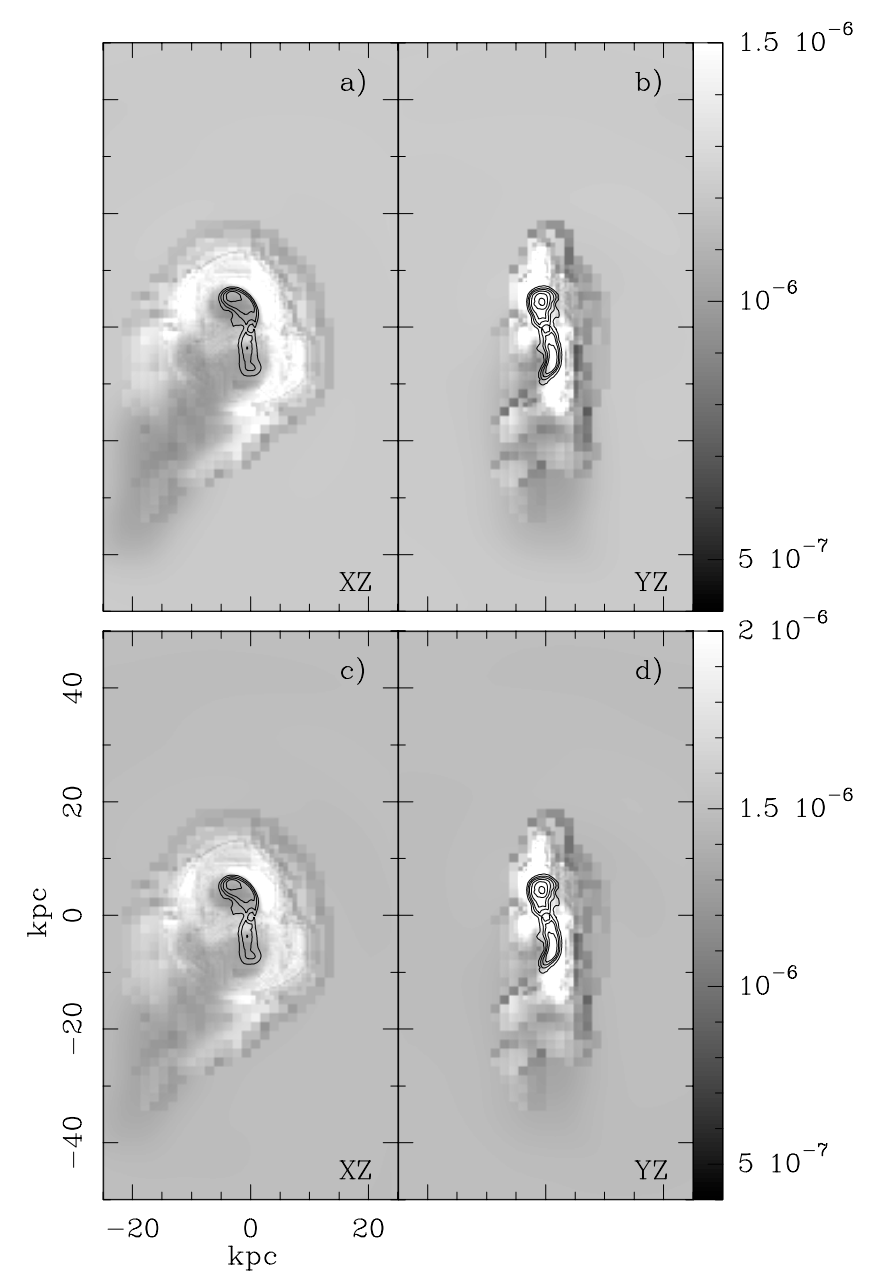

Fig. 3. X-ray emission maps generated from model I. Panels a) and b) show, in gray-scale, the emission in the soft energy band $(0.3-1.8 \mathrm{keV})$ for the $x z$ and $y z$ projection, respectively. The same projections, except for the hard band (1.8-7 keV), are shown in panels c) and d). Each map is overlayed with average temperature contours in the $[2-8] \times 10^{7} \mathrm{~K}$ range. The vertical bars to the right of panels b) and d) represent the linear gray scale of the X-ray emission, in units of $\mathrm{erg} \mathrm{s}^{-1} \mathrm{~cm}^{-2} \mathrm{sr}^{-1}$. All maps correspond to an integration time of $121 \mathrm{Myr}$. Notice that darker areas correspond to lower emission regions.

the X-ray map obtained by integrating the emission coefficient along the $y$-axis ( $x z$ projection), while the upper right panel displays the map obtained when the integration is carried out along the $x$-axis ( $y z$ projection). The top two panels correspond to the X-ray emission in the soft energy band $(0.3-1.8 \mathrm{keV})$ for an evolution time of $121 \mathrm{Myr}$. The same time-frame is shown in the bottom two panels (i.e. for the two projections), but for the hard energy band (1.8-7 keV) X-ray emission. In order to compare the X-ray emission with the morphology of the jets, all gray-scale X-ray maps are overlayed with contours of the corresponding temperature maps (averaged along the same linesof-sight).

From the simulated X-ray maps, we see in both Figs. 3a and $3 \mathrm{c}$ (the $x z$ projection) that the maps show a bright X-ray filament characterized by an overall bow-shock shape. The wake, observed in the density distribution maps, presents X-ray emission which is lower than the one of the intra-cluster medium 


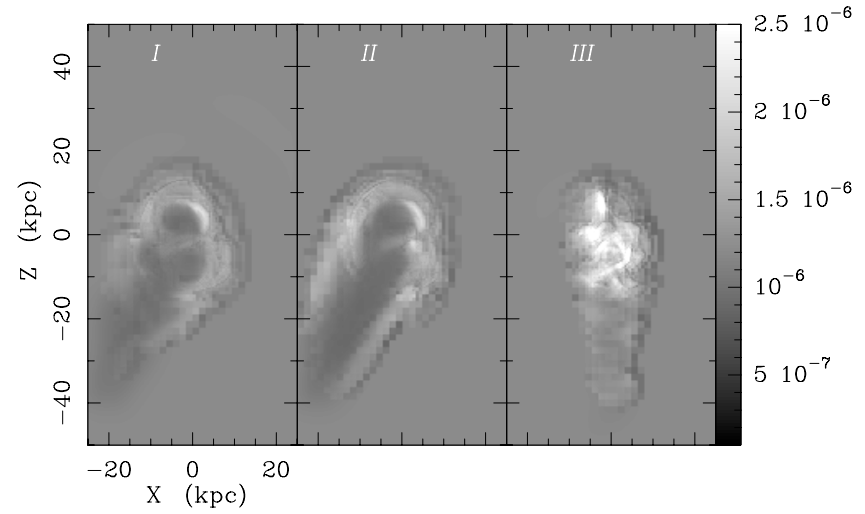

Fig. 4. X-ray emission maps in the soft energy band (0.3-1.8 keV), for models I, II, and III (left, middle, and right panels, respectively). The $\mathrm{X}$-ray emission from Model II (with a precession period five times shorter than model I) looks similar to that of model I. The X-ray emission from model III is quite different from the other two, showing several bright, aligned filaments. The three maps correspond to an integration time of 121 Myr. The linear gray scale of the X-ray emission is represented by the vertical bar on the extreme right, in units of $\mathrm{erg} \mathrm{s}^{-1} \mathrm{~cm}^{-2} \mathrm{sr}^{-1}$. Notice that darker areas correspond to lower emission regions.

emission. The X-ray emission from the lobe jets is also low. In contrast, in Figs. $3 \mathrm{~b}$ and $3 \mathrm{~d}$ (the $y z$ projection), the bright bow shock filament is now observed as a more linear structure superposed on the jets rather than to one side. This fact shows that the orientation of the flow with respect to the observer has a strong effect on the predicted X-ray emission maps. In other astrophysical scenarios, such as in stellar winds and supernova remnants, the importance of projection effects was pointed out by Hnatyk \& Petruk (1999), Petruk (2001), and Velázquez et al. (2004b) . In our case, we show that in the $y z$ projection the cavities (at the lobe jet positions) and wake (observed in the $x z$ projection) practically disappear, because they are hidden by the bright emission from the bow shock structure, which is located along the same lines of sight.

We have verified how sensitive model I is with respect to the adopted parameters. Figure 4 shows the $\mathrm{X}$-ray emission for models I, II, and III in the soft energy band $(0.3-1.8 \mathrm{keV})$. The $\mathrm{X}$-ray emissions from models I and II are very similar, even though the precession period is five times faster in model II. However, in model III the morphology of the X-ray emission changes drastically as a result of having the plane-parallel wind moving exclusively in the $z$ direction. The $\mathrm{X}$-ray emission of model III can be described as being made of several centrally positioned filaments surrounded by a lower X-ray emission gas. The morphology shown in model III looks similar to the one observed in radio galaxy jets such as 3C 294 (Crawford et al. 2003).

For each map of models I, II, and III, we have derived the corresponding energy distribution, or synthetic spectrum, in the $0.3-7.0 \mathrm{keV}$ interval, which are plotted in Fig. 5. These take the absorption by an interstellar absorption into account corresponding to a hydrogen column density of $N_{\mathrm{H}}=2 \times 10^{20} \mathrm{~cm}^{-2}$. The three spectra are identical and show spectral features that

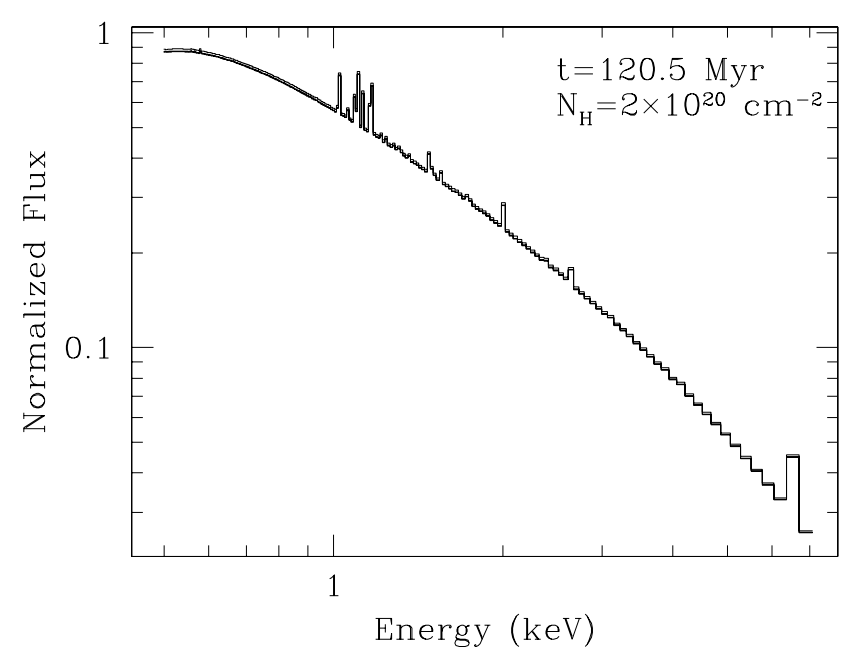

Fig. 5. Normalized synthetic spectra from models I, II and III. These spectra correspond to an evolution time of $121 \mathrm{Myr}$ and include absorption by a $N_{\mathrm{H}}=2 \times 10^{20} \mathrm{~cm}^{-2}$ hydrogen column density.

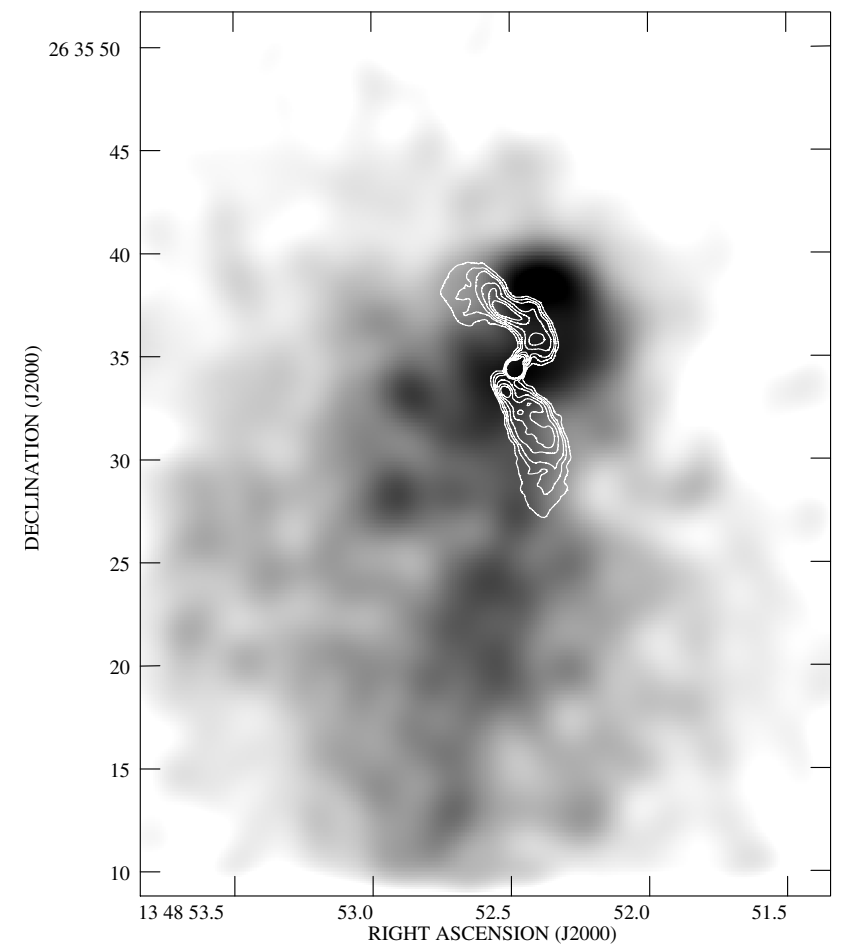

Fig. 6. Overlay of the radio-continuum emission at $8.3 \mathrm{GHz}$ (in white contours, Ge \& Owen 1993) with X-ray diffuse emission from A1795 cluster (in greyscale, Fabian et al. 2001). The contours corresponds to the $0.1,0.2,0.3,0.5$ and 1. mJy beam $^{-1}$ levels. Dark areas in greyscale correspond to strong enhancement in X-ray emission.

were reported by others, such as the presence of some Fe lines close to $1 \mathrm{keV}$ (see Peterson 2003).

\section{Analysis of results and comparison with observations}

\subsection{Radio jet in the Abell 1795 galaxy cluster}

As a prototype of filamentary structure in X-ray emission, we have the Abell 1795 cluster case. Abell 1795 (hereafter A 1795) 
is a rich cluster of galaxies, which has been studied at optical, radio, and X-ray wavelengths. Figure 6 shows an overlay of the X-ray emission (in greys) from this cluster (obtained with CHANDRA satellite, Fabian et al. 2001), and radio-continuum at $8.3 \mathrm{GHz}$ of the central region (white contours, Ge \& Owen 1993).

Radio observations of the central region of A 1795 show a jet-like emission from the bipolar radio source 4C 26.42 with an "S" shape (see white contours in Fig. 6). The projected combined size ${ }^{2}$ is $\sim 5.6 \times 16.2 \mathrm{kpc}$ (van Breugel et al. 1984 and Ge \& Owen 1993). In X-rays, high resolution images from CHANDRA show the presence of a long $(\sim 80 \mathrm{kpc})$ knotty filament in the North-South direction (see Fig. 6). This filament is formed by hot gas with temperatures on the order of $2.7 \mathrm{keV}$ (by a three ray color analysis, Fabian et al. 2001). This large scale filament coincides positionally rather well with the elongated optical $\mathrm{H} \alpha+[\mathrm{NII}]$ emission line feature previously observed by Cowie et al. (1983). Ettori et al. (2002) carried out a similar study by focusing on the characteristics of the ICM of A 1795. From spectral analyses, they find that the temperature of the ICM is in the [3.5-4.5] keV range, higher than the X-ray filament temperature reported by Fabian et al. (2001).

\subsection{Comparison between observations and main numerical results}

The main results obtained from our numerical simulations are:

- By means of integrated temperature maps (Fig. 2), our model describe the shape of the radio-jets emanating from the central galaxy of A 1795 quite well, giving physical dimensions for the lobes of $\sim 10 \mathrm{kpc}$, which is in quantitative agreement with the radio image obtained by van Breugel et al. (1984).

- The wake left behind by the interaction of the jets with the plane-parallel wind, as observed in the density maps (Fig. 1), resembles the morphology of the observed X-ray filament of A 1795 (Fig. 6). However, this "wake" cannot be the origin for the bright filament in A1795 because its $\mathrm{X}$-ray emission is lower than the ICM emission.

- Bright X-ray emission filaments were obtained from our three models (specially in the soft X-ray band). Simulated X-ray emission maps show a "bow shock" feature (in the $x z$ projection), which is a consequence of the jet-plane parallel wind interaction (see the left panels of Fig. 3 and panels I and II of Fig. 4). This "bow shock" feature is seen as a long, straight filament in the yz projection maps (Fig. 3). By direct comparison with the observational results of Fabian et al. (2001) and Ettori et al. (2002), models I and II describe the X-ray emission of A 1795 better because the simulated X-ray emission maps exhibit a single bright filament (compare $y z$ projection of Figs. 3 with 6 ). The simulated maps from model III can be employed to explain the X-ray morphology in 3C 294 (Crawford et al. 2003).

- The simulated, bright X-ray filaments correspond to emission from gas with temperatures a factor $\sim 1.4$ higher than

\footnotetext{
${ }^{2}$ We used $H_{0}=70 \mathrm{~km} \mathrm{~s}^{-1} \mathrm{Mpc}^{-1}$, finding that $1^{\prime \prime}=1.31 \mathrm{kpc}$.
}

the ICM temperature. This result differs from the observational result $T_{\text {filament }} / T_{\mathrm{ICM}}=0.66$ obtained by Fabian et al. (2001) and Ettori et al. (2002). A discussion of this discrepancy follows.

- Synthetic spectra from the three models are very similar to each other, and are also in good qualitative agreement with the observations.

\section{Discussion}

We have computed models of a precessing, bipolar jet ejected by a radio galaxy in motion with respect to the surrounding intra-cluster environment. Our models were computed for parameters that are relevant for the jets and X-ray filament associated with the 4C 26.42 galaxy in the A 1795 cluster. Previous works (Reynolds et al. 2001; Zanni et al. 2003) that have studied jet-ICM interaction predict a shell-like morphology (for supersonic cocoon expansion regime) or partial shell-like shape (for subsonic cocoon expansion regime) in X-ray emission. Furthermore, the predicted X-ray emission is symmetric. The main difference between these models and our work is that the jet source is moving into the ICM, thus generating an enhancement in the part of cocoon located in the direction of the incoming ICM wind.

From an analysis of our numerical results, we conclude that a precessing jet interacting with a plane-parallel ambient wind qualitatively explains many of the morphological features observed in A 1795. A jet ejected from a source moving within the intra-cluster environment with the velocity of 4C 26.42 leaves behind a wake with a similar morphology to the one of the X-ray filament associated with this object (Fabian et al. 2001). The jet precession at the same time produces lobes with a radio morphology similar to the one of the radio jets ejected by 4C 26.42 (van Breugel et al. 1984; Ge \& Owen 1993). Besides, the jet precession has a secondary effect by producing a knotty wake structure. To this extent, our numerical models are successful at reproducing the characteristics of the jet $+\mathrm{X}$ ray filament system associated with this galaxy.

However, Ettori et al. (2002) and Fabian et al. (2001) find that the X-ray spectrum of the A 1795 filament and that its surrounding intra-cluster medium implies two different temperatures. Fabian et al. (2001) find the temperature of the filament might be lower than $2.7 \mathrm{keV}$, while Ettori et al. (2002) give a temperature between 3.5 and $4.5 \mathrm{keV}$. Rodríguez-Martínez et al. (2006) will present a new analysis using all archived CHANDRA data of A 1795.

Our non-radiative models clearly do not reproduce this effect, as the temperatures obtained for the gas within the wake are always higher than the temperature of the surrounding, unperturbed intra-cluster medium. However, it might be possible to reconcile our model with the X-ray observations of A 1795 by including thermal conduction and/or turbulent mixing of the hot, post-bow shock gas with material in a cold phase (of $\sim 10^{4} \mathrm{~K}$ ) belonging in the past to the interstellar medium of the central galaxy. In order to include such effects together with a radiative cooling, a much higher resolution simulation would be necessary. 
Notwithstanding this discrepancy, it is interesting that such a simple model reproduces the morphology of bright X-ray filaments similar to the one observed in A 1795 and other clusters. Structures with similar sizes to the observed X-ray filament can be generated in our model. Furthermore, observed spectral features are present in our synthetic spectra, such as the emission by very high ionization Fe lines. Of the three models presented in this work, we consider both models I and II as acceptable, with model III used only to explore the impact of varying the incidence angle of the wind.

Acknowledgements. We would like to thank the referee for many helpful suggestions. The authors acknowledge support from CONACyT grants 36572-E, 40096-F, 41320-E, 43103-F, and DGAPA-UNAM grants IN118601, IN112602 and IN113605. We are grateful to Andy C. Fabian and Gregory Taylor for the kind help with their radio and X-rays images of A1795. We thank Israel Díaz for maintaining our Linux servers, where all the numerical simulations were carried out.

\section{References}

Anders, E., \& Grevesse, N. 1989, Geochim. Cosmochim. Acta, 53, 197

Condon, J. J., \& Mitchell, K. J. 1984, ApJ, 176, 472

Cox, C. I., Gull, S. F., \& Scheuer, P. A. G. 1991, MNRAS, 252, 558

Cowie, L. L., Hu, E. M., Jenkins, E. B., \& York, D. G. 1983, ApJ, 272, 29

Crawford, C. S., Fabian, A. C., Sanders, J. S., \& Ettori, S. 2003, NewAR, 47, 239

Dere, K. P., Landi, E., Young, P. R., \& Del Zanna, G. 2001, ApJS, 134,331

Ettori, S., Fabian, A. C., Allen, A. W., \& Johnstone, R. M. 2002, MNRAS, 331, 635
Fabian, A. C., Sanders, J. S., Ettori, S., et al. 2001, MNRAS, 321, L-33

Ferrari, A. 1998, ARA\&A, 36, 539

Ge, J. P., \& Owen, F. N. 1993, AJ, 105, 778

Hill, J. M., Hintzen, P., Oegerle, W. R., et al. 1988, ApJ, 332, L23

Hnatyk, B., \& Petruk, O. 1999, A\&A, 344, 295

Kellerman, K., \& Owen, F. 1988, in Galactic and extragalactic radio astronomy, 2nd edition (Berlin, New York: Springer-Verlag), 563

Masciadri, E., de Gouveia Dal Pino, E. M., Raga, A. C., \& Noriega-Crespo, A. 2002, ApJ, 580, 950

Morrison, R., \& McCammon, D. 1983, ApJ, 270, 119

Mazzotta, P., Mazzitelli, G., Colafranscesco, S., \& Vittorio, N. 1998, A\&As, 133, 403

Oegerle, W. R., \& Hill, J. M. 1994, AJ, 107, 857

Peterson, J. R., Kahn, S. M., Paerels, F. B. S., et al. 2003, ApJ, 590, 207

Petruk, O. 2001, A\&A, 371, 267

Pietrini, P., \& Torricelli-Ciamponi, G. 1992, ApJ, 401, 60

Raga, A. C., Navarro-González, R., \& Villagrán-Muniz, M. 2000, Rev. Mex. Astron. Astrofis., 36, 67

Raga, A. C., Velázquez, P. F., Cantó, J., Masciadri, E., \& Rodríguez, L. F. 2001, ApJ, 559L, 33

Reynolds, C. S., Heinz, S., \& Begelman, M. C. 2001, ApJ, 549, 179

Rizza, E., Loken, C., Bliton, M., et al. 2000, ApJ, 119, 21

Rodríguez-Martínez, M., Binette, L., Velázquez, P. F., et al. 2005, in preparation

Torricelli-Ciamponi, G., \& Pietrini, P. 1993, ApJ, 406, 36

van Breugel, W., Heckman, T., \& Miley, G. 1984, ApJ, 276, 79

van Leer, B. 1982, ICASE Rep, 82

Velázquez, P. F., Riera, A., \& Raga, A. C. 2004a, A\&A, 419, 991

Velázquez, P. F., Martinell, J., Raga, A. C., \& Giacani, E. B. 2004b, ApJ, 601, 885

Young, P. R., Del Zanna, G., Landi, E., et al. 2003, ApJS, 144, 135

Zaninetti, L., \& van Horne, H. M. 1988, A\&A, 189, 45

Zanni, C., Bodo, G., Rossi, P., et al. 2003, A\&A, 402, 949 